\title{
ESTRATEGIA DE EDUCACIÓN EN SALUD Y AMBIENTES SALUDABLES, PARA NIÑAS, NIÑOS Y ADOLESGENTES BAJO PROTECGIÓN
}

\section{HEALTH EDUCATION AND HEALTHY ENVIRONMENTS STRATEGY FOR CHILDREN AND ADOLESGENTS UNDER PROTECTION}

\author{
Paula Andrea Ávila-Vélez ${ }^{1}$ \\ Luisa Fernanda Leiva-Benavides ${ }^{2}$ \\ Johanna Constanza Bueno-Gutiérrez ${ }^{3}$ \\ María Fernanda Gámez-Peralta ${ }^{4}$
}

Fecha de Recepción: 16 de junio de 2016

Fecha de Aceptación: 18 de diciembre de 2016

Citar como:

Ávila-Vélez P, Leiva-Benavides L, Bueno-Gutiérrez J, Gámez-Peralta M. (2016). Estrategia de educación en salud y ambientes saludables, para niñas, niños y adolescentes bajo protección. Revista Movimiento Científico 10(2): 41-55.

Ávila-Vélez P, Leiva-Benavides L, Bueno-Gutiérrez J, Gámez-Peralta M. (2016). Estrategia de educación en salud y ambientes saludables, para niñas, niños y adolescentes bajo protección. Revista Movimiento Científico [en línea] 2016, [fecha de consulta: dd// mm/aaaa]; 10(2): 41-55. Disponible desde: http://revistas.iberoamericana.edu.co/index.php/Rmcientifico/issue/archive.

\section{RESUMEN}

Introducción: la infancia es considerada un mundo social que fundamentado a partir de diferentes roles creados desde su participación activa en la comunidad; para esto, es importante favorecer procesos de desarrollo motor con el fin de potenciar habilidades y desarrollar intereses en la adherencia a estilos de vida saludable. Objetivo: diseñar una estrategia de educación para la salud y ambientes saludables desde fisioterapia para niños, niñas y adolescentes en situación de protección en el contexto colombiano. Materiales y Método: se realizó un estudio descriptivo de desarrollo tecnológico (experimental) respondiendo a los postulados de la OCDE, y Hernández, Fernández y Baptista. La investigación específicamente describe el desarrollo de un nuevo procedimiento para favorecer estilos de vida saludable en niños, niñas y adolescentes. Resultados: se encontró una restricción en la participación debido al contexto en el que permanecen; sin embargo, fue posible reconocer un alto interés de los niños hacia la actividad física con prevalencia en actividades como fútbol y baile, a partir de lo cual surge el diseño de actividades que se configuran en una estrategia centrada en la educación en salud y prevención primaria, según grupo etario. Las actividades

Fisioterapeuta. Institución Escuela Colombiana de Rehabilitación.

Fisioterapeuta. Institución Escuela Colombiana de Rehabilitación. Correo electrónico: luisaleiva@ecr.edu.co

Fisioterapeuta. Institución Escuela Colombiana de Rehabilitación. Estudiante de Psicología. Universidad Externado de Colombia.

Fisioterapeuta. Institución Escuela Colombiana de Rehabilitación. 
proponen impactar motricidad fina, gruesa, esquema corporal, así como el reconocimiento del autocuidado y el balance nutricional. Conclusiones: Se reconoce la necesidad y pertinencia de la participación del fisioterapeuta en la planeación de actividades de educación en salud y prevención primaria para la infancia y adolescencia a lo largo del ciclo vital de población bajo protección.

Palabras clave: Actividad motora, Niño, Adolescente, Promoción de la salud, Prevención primaria, Estilo de vida.

\section{ABSTRACT}

Background: childhood in considered a social world that is based in different roles created from its active participation in the community, for this, its important to favour processes of motor development with the purpose of boosting abilities and develop interests in the adherence of healthy life styles. Objective: to design a strategy of education for health and healthy environments from Physical Therapy for children and adolescents in a situation of protection in the Colombian context. Materials and Methods: A descriptive study of technological development (experimental) was realized to respond to the OECD and Hernández, Fernández and Baptista postulates. The investigation specifically describes the development of a new process to promote healthy lifestyles in children and adolescents. Results: It was found a restriction due to the environment they remain. However, it was possible to recognize a high interest from the children towards physical activity prevailing in activities such a dance and football, from this, arises the design of activities that are configured in a strategy centred to health education and primary prevention, according to age group. The activities propose to impact fine and gross motricity body layout as well as the recognition of self care and nutritional balance. Conclusions: It is recognized the necessity and relevance of the participation of the physiotherapist in the planning of activities of health education and primary prevention for childhood and adolescence throughout the life cycle of the population under protection.

Keywords: Motor activity, Child, Adolescent, Health promotion, Primary prevention, Life style.

\section{INTRODUCCIÓN}

La infancia es considerada un mundo social donde los roles se fundamentan a partir de diferentes componentes, dentro de ellos se puede encontrar: la personalidad, las capacidades del niño, las relaciones psicosociales, las actitudes y competencias del educador (en el caso de niños escolarizados) y las exigencias y experiencias según su entorno (Presutti, s.f.).

La adolescencia es un periodo de crecimiento y desarrollo humano que da paso a la edad adulta; de acuerdo al Código de la Infancia y la Adolescencia colombiano, se diferencia de la infancia entendiendo que niño o niña hace referencia a personas entre los 0 y los 12 años y adolescente a personas entre 12 y 18 años de edad (Ley 1098, 2006). Los niños, niñas y adolescentes cumplen diferentes roles en la sociedad y debe garantizarse su participación activa en los diferentes escenarios (Bellamy, 2005).
En cuanto al desarrollo en la primera infancia, según la OMS (2013) el desarrollo infantil es un proceso dinámico, por el cual los niños progresan desde una etapa de dependencia en sus áreas de funcionamiento, hacia una gradual independencia en la segunda infancia (edad escolar), la adolescencia y la adultez; en tal proceso, logran habilidades en diferentes ámbitos como el sensorial-motor, cognitivo, comunicacional y socio-emocional.

En los primeros años de vida se ven expuestos a factores de riesgo como la pobreza, el estigma, la discriminación, mala interacción con sus cuidadores, la institucionalización, la violencia, el abuso, el abandono, y el acceso limitado a programas y servicios, lo cual puede afectar significativamente su supervivencia y desarrollo. Específicamente en el contexto colombiano estos factores conllevan a vincular a los niños, niñas y 
adolescentes a programas de protección, para que el gobierno garantice sus derechos. Según la UNICEF (2005), las cifras de niños y adolescentes en situación de protección han aumentado anualmente.

De acuerdo al Código de la Infancia y la Adolescencia en Colombia, esta población cuenta con el derecho a la generación de condiciones que aseguren desde su nacimiento el cuidado, la protección, la alimentación nutritiva y equilibrada, el acceso a los servicios de salud, educación, vestuario adecuado, a la recreación y la vivienda segura; por tal razón, los menores de edad se convierten en sujetos de derechos y son considerados como seres en desarrollo; adicional a esto, son etapas del ciclo vital en la cuales se establecen bases importantes para el desarrollo cognitivo, emocional y social del ser humano (Ley 1098, 2006).

Dentro del acceso a servicios de salud en instituciones dedicadas a la protección de niños, niñas y adolescentes, se ha vinculado a los fisioterapeutas como protagonistas de la identificación y propuesta de acciones de educación para la salud y ambientes saludables en contextos no clínicos; esto conlleva a estructurar estrategias profesionales que favorezcan el cumplimiento de derechos en cuanto a la participación en entornos de vida saludable desde la perspectiva del movimiento corporal humano.

A partir de lo anterior se considera importante que cada acción propuesta desde la Fisioterapia esté dirigida a favorecer el aprovechamiento del tiempo libre a través de la actividad física, con el fin de dar uso constructivo de este para el enriquecimiento personal y el disfrute de la vida en forma individual y colectiva. Diferentes profesiones abordan la realización de actividad física; sin embargo, como se mencionó, es interés del presente artículo la acción desde la Fisioterapia, que en sus áreas de desempeño profesional propone acciones hacia el individuo, la familia y la comunidad en sus diferentes entornos (Ley 528, 1999).

Entendiendo que las acciones de la Fisioterapia en el contexto de protección se realizan tomando elementos conceptuales de la promoción y la prevención, se debe reconocer que la promoción de la salud consiste en proporcionar los medios necesarios para mejorar la salud y ejercer control sobre la misma, logrando alcanzar un bienestar físico, mental y social, ya sea de un grupo o un individuo; para este documento se tienen en cuenta acciones enfocadas en la educación de la salud y entornos saludables desde los ejes temáticos de actividad física, alimentación e higiene (Vargas, Villegas, Sánchez y Holthuis, 2003).

En este sentido, la prevención se refiere a las medidas destinadas a evitar la aparición de la enfermedad, detener su avance y reducir sus consecuencias, se clasifica en tres niveles: prevención primaria, secundaria y terciaria; siendo relevante para el presente documento la prevención primaria, la cual tiene como objetivo disminuir la incidencia de la enfermedad por medio del control del factor causal y predisponente (Vignolo, Vacarezza, Álvarez y Sosa, 2011).

Desde la Fisioterapia, se reconoce la actividad física como un medio clave para modificación de estilos de vida; esto es aceptado por la OMS (2010), quienes refieren que la actividad física está asociada con efectos psicológicos beneficiosos en los jóvenes gracias a un mejor control de la ansiedad y la depresión; también es importante reconocer que está asociada con la adquisición de una imagen corporal más saludable, reforzando la percepción del sujeto sobre sí mismo; adicionalmente, tiene efectos sobre los diferentes sistemas, como el sistema locomotor, cardiovascular y neuromuscular en cuanto a la optimización de su funcionamiento. Por otra parte, realizar algún tipo de actividad física evita la adquisición de hábitos no saludables como el consumo de alcohol, cigarrillo o drogas y junto a esto suelen demostrar un mejor rendimiento escolar.

La OMS (2010) recomienda que los niños deben realizar actividad física con el fin de mantener o mejorar la salud, principalmente cuando se encuentran en las edades entre 5 y 17 años; que se fomenta a través de juegos, deportes, desplazamientos, recreación, educación física o ejercicios programados en los entornos familiar, escolar y comunitario; recomendando que se invierta como mínimo 60 minutos diarios en actividades de moderada a vigorosa intensidad. 
De igual forma, según Zamora y Laclé (2012), la actividad física realizada a dicha intensidad y con un gasto energético mayor a las $300 \mathrm{kcal} / \mathrm{h}$ en más de 4h/semana puede mejorar la calidad de vida de los niños.

El ejercicio presentado en este artículo parte de un proceso de práctica formativa que se realizó en la segunda rotación del segundo periodo académico del año 2015, por parte de estudiantes de Fisioterapia de la Escuela Colombiana de Rehabilitación (ECR), y se proyectó desde un proceso de evaluación realizado con niños entre 1 a 14 años, que corresponde a la franja de edad de la población vinculada a una institución para el cuidado de niños, niñas y adolescentes en la ciudad de Bogotá.

Se debe tener en cuenta que la población de interés se encuentra en situación de protección debido a que sus derechos han sido vulnerados y con factores de riesgo que en consecuencia afectan su desarrollo psicomotor y limitan un adecuado establecimiento de hábitos y estilos de vida saludables; a partir de esto, se plantea como principal factor desencadenante el contexto donde se desenvuelven antes y durante la estancia en la institución y la carencia de la red de apoyo, específicamente la familia nuclear, lo cual limita la adquisición de bases que se transmiten de padres a hijos, entre ellos: hábitos y estilos de vida esenciales que tendrán grandes compensaciones a nivel del bienestar físico, psicológico y social en el niño.

A partir de esto se plantea como objetivo general diseñar una estrategia de educación en salud y ambientes saludables para niños, niñas y adolescentes en situación de protección en el contexto colombiano, a partir del planteamiento de acciones estratégicas orientadas a la promoción de la salud y prevención de la enfermedad acordes a la edad.

\section{MATERIALES Y MÉTODOS}

En el presente estudio se realizó un proceso descriptivo que permitió, a partir de las características de una población, especificar unas estrategias de acompañamiento desde la Fisioterapia; este alcance es consistente con los tipos de estudios propuestos por Hernández, Fernández y Baptista (2014), quienes mencionan que estas investigaciones describen "fenómenos, situaciones, contextos y sucesos; esto es, detallar cómo son y se manifiestan” (p.92).

Ahora bien, dentro de la amplia gama de estudios descriptivos, este estudio corresponde metodológicamente a un desarrollo tecnológico, también denominado en el Manual de Frascati de la OCDE (2003) como proyecto de desarrollo experimental en la medida en que establece "nuevos procedimientos, sistemas y servicios, o a mejorar considerablemente los ya existentes" (p. 2).

El desarrollo de esta investigación surgió desde de una práctica formativa del programa de Fisioterapia de la Escuela Colombiana de Rehabilitación. El proceso investigativo se realizó de forma censal con un total de 79 niños, niñas y adolescentes (43 mujeres y 36 hombres), con edades entre 1 a 14 años en situación de protección en una institución ubicada en la Localidad de Suba. Las acciones de evaluación se realizaron como parte de los procesos de atención rutinaria de la institución (desde promoción y prevención), por esto no se hizo necesaria la aplicación de consentimientos $\mathrm{y}$ asentimientos informados.

Para la identificación de las características de la población se implementaron dos instrumentos de recolección de información: la Escala Abreviada del Desarrollo (EAD) y el Physical Activity Questionnaire for Adolescents (PAQ-A) y el Physical Activity Questionnaire for Children (PAQ-C). La EAD evalúa como variables según áreas: a) motricidad gruesa, b) motricidad fina adaptativa, c) audición/lenguaje y d) personalsocial, siendo aspectos importantes para el desarrollo psicomotor; esta escala se califica en función de 4 "franjas" en donde "alerta" es la peor condición; seguida por "medio" y "medio alto", como condiciones intermedias; y "alto" como mejor condición; la EAD fue aplicada en niños y niñas específicamente de 1 año de edad (Muñoz, Zapata-Ossa y Pérez-Tenorio, 2013).

El PAQ-A específico para adolescentes (MartínezGómez et al, 2009) y PAQ-C, específico para niños 
(Herazo-Beltrán y Domínguez-Anaya, 2012) permiten medir las actividades realizadas en el tiempo libre en diferentes contextos de la vida diaria, intensidad y frecuencia en la semana anterior de la aplicación del instrumento. Estos instrumentos utilizan una escala tipo Likert de 1 a 5 ; sin embargo, para documentar las actividades en niños entre 2 y 5 años, el PAQ - C se aplicó acompañado de ilustraciones.

Para la delimitación de las acciones que componen la estrategia, a presentar en el siguiente apartado, se realizó un proceso documental a partir de la búsqueda de artículos, por medio de la combinación de palabras clave tales como: [actividad física]; [Fisioterapia en comunidad]; [Physicalactivity]; [actividad física en niños]; [hábitos de vida saludable]; [actividad física]; [adolescente]; [cuestionario]; [PAQ-A]; [validación]; [Escala Abreviada del Desarrollo]; [PAQ-C]; [PhysicalTherapy]; [Children]; [promoción y prevención]; [Fisioterapia] en las bases de datos Bireme, Medline, Scielo, Elsevier y PubMed.

Una vez sistematizados los textos a partir de los cuales se obtuvo la información de referencia para la constitución de las acciones, se aplicó la técnica de "Corte y clasificación”, que según Hernández, Fernández y Baptista (2014) consiste en "identificar expresiones, pasajes o segmentos que parecen importantes para el planteamiento y luego juntarlos conceptualmente" (p. 43). Esto permitió consolidar los referentes a partir de los cuales se realizó el diseño de las acciones que se proponen a continuación.

\section{RESULTADOS}

El desarrollo de la propuesta denominada "El movimiento es vida" inició por medio de la caracterización de la población e identificación del entorno a través de la implementación de instrumentos de evaluación específicos de acuerdo al rango etario.

En cuanto a los hallazgos de la EAD, en el área de motricidad gruesa se encontró que los niños se categorizan entre la franja de "alerta" y "medio", en el área de motricidad fina y adaptativa en la franja "medio", en el área de audición y lenguaje en la franja de "alerta” y por último en el área personal - social en la franja "medio"; los resultados de cada categoría se constituyen en un total sumario que permite categorizar a los niños en la franja "alerta", esto indica que su desarrollo integral no se encuentra acorde a su edad cronológica.

Con los cuestionarios PAQ - C y PAQ - A se indagó acerca de las actividades realizadas con mayor frecuencia, teniendo en cuenta que el reconocer estas actividades genera un punto de inicio para programar actividades conocidas por los niños. Dentro de las actividades identificadas se destacaron el fútbol, baloncesto, baile, saltar lazo y carrera, reportando que se realizaban con mayor frecuencia en las clases de educación física de la población escolarizada -entendiendo que en la institución se encuentra población en proceso de escolarización-, por otra parte, practican actividades de juego libre con una frecuencia de 3 días por semana a una intensidad moderada.

En general dentro del proceso de evaluación, se encontró una restricción en la participación debido al contexto donde permanecen los niños de la institución, teniendo en cuenta la poca accesibilidad a diferentes entornos. Adicionalmente fue posible establecer un alto interés de los niños hacia la actividad física, sin embargo, existen diferencias en cuanto al interés de actividades específicas.

Por medio de la información recolectada en este proceso, los hallazgos más importantes refieren que la población de niños tiene la necesidad de estimular y garantizar el desarrollo integral en la etapa de educación inicial y que el aporte sea continuo en el ciclo vital, por lo que es importante instaurar estilos de vida saludable en la cotidianidad de esta población de acuerdo al rango etario y teniendo en cuenta el contexto.

Con base en lo anterior, se propuso una estrategia compuesta por actividades de educación para la salud y ambientes saludables desde la Fisioterapia acorde a las edades de los niños, niñas y adolescentes, entendiendo estos factores como elementos de importancia para la interacción en los diferentes entornos. 
A continuación, se describen dichas actividades por rangos de edad y de acuerdo al alcance ya sea de promoción de la salud desde educación para la salud y ambientes saludables, y de prevención primaria de eventos en salud que según Cigarroa, Sarqui y Zapata-Lamana (2016) pueden ser, dentro de las más prevalentes: el aumento de peso corporal, baja competencia motriz y el desarrollo de enfermedades crónicas no transmisibles en etapas posteriores de la vida.

Es pertinente precisar que una misma actividad puede impactar en más de un componente evaluado a partir de los instrumentos ya mencionados, por tanto, las actividades no se organizarán en función de los atributos impactados, sino desde el objetivo de la Fisioterapia.

Las propuestas de actividades se contemplan con un nombre descrito en la fila 2 de las tablas 1 a 13, el cual cumple una función de etiqueta, de modo que puedan ser reconocidas por los niños a partir de una denominación conocida y significativa; también se presenta el objetivo principal que permite dar orientación a la actividad, acompañado de la descripción del proceso y finalmente los resultados después de su aplicación.

\section{ACTIVIDADES PARA NIÑOS ENTRE 1 A 5 AÑOS}

Tabla 1. Actividad "Los juegos de las manos"

\begin{tabular}{ll}
\hline ALCANCE & Promoción y prevención \\
\hline ACTIVIDAD & Los juegos de las manos \\
\hline OBJETIVO & Potencializar la motricidad fina y gruesa en las actividades de higiene personal. \\
\hline DESCRIPCIÓN DE & La actividad se basa en hacer conciencia del uso de las manos en todas las actividades y sobretodo \\
LA ACTIVIDAD & de lo importante que es tenerlas limpias para las mismas, por eso las personas a cargo tendrán \\
& $\begin{array}{l}\text { apoyos visuales con imágenes sobre "para qué usamos nuestras manos" y la forma adecuada de } \\
\text { realizar el aseo de las mismas, se finaliza la actividad con conclusiones de los niños encaminadas } \\
\text { al aseo de las manos tanto antes y después de comer, jugar, ir al baño, etc. }\end{array}$ \\
\hline
\end{tabular}

RESULTADOS ESPERADOS Se pretende potenciar habilidades de motricidad fina y gruesa a partir de actividades de autocuidado como lavado de manos y cara reforzando destrezas al desempeñarlas.

Fuente: elaboración propia (2016)

Tabla 2. Actividad "Fiesta Hawaiana"

\begin{tabular}{ll}
\hline ALCANCE & Promoción \\
\hline ACTIVIDAD & Fiesta Hawaiana \\
\hline OBJETIVO & $\begin{array}{l}\text { Reforzar el desarrollo de la motricidad fina, coordinación ojo-mano, coordinación global y patrones } \\
\text { básicos de movimiento para potencializar las habilidades de dibujo, escritura y manipulación de } \\
\text { alimentos por medio de actividades artísticas y juegos competitivos. }\end{array}$ \\
\hline DESCRIPCIÓN DE & Se divide en 2 partes, en la primera los niños realizan elementos de sus propios disfraces, donde \\
LA ACTIVIDAD & utilizan la motricidad fina, para hacer collares, manillas y adornar bolsas de Halloween (o un elemento \\
& similar) marcándolas con sus nombres. En el segundo realizan actividades, en competencia como: \\
& bailar aprendiendo coreografías y siguiendo pasos indicados, correr, jugar limbo, hacer cestas, realizar \\
& lanzamientos golpeando un objetivo, con el fin de potenciar el desarrollo de patrones motores. \\
\hline RESULTADOS ESPERADOS & $\begin{array}{l}\text { Se logró mejoría en la motricidad fina, identificación de figuras, colores y seguimiento de } \\
\text { instrucciones, además del trabajo en equipo. }\end{array}$ \\
\hline
\end{tabular}

Fuente: elaboración propia (2016) 
Tabla 3. "Enseñarle a Pablito"

\begin{tabular}{ll}
\hline ALCANCE & Promoción y prevención \\
\hline ACTIVIDAD & Enseñarle a Pablito \\
\hline OBJETIVO & $\begin{array}{l}\text { Reconocer los procesos motrices alrededor de los hábitos de autocuidado y autonomía } \\
\text { enfocándose en la enseñanza de ir al baño, vestirse, bañarse y sonarse con el fin de que lo } \\
\text { asuman como hábito de vida. }\end{array}$ \\
\hline DESCRIPCIÓN DE & Se representan situaciones como: jugar, comer, ir al baño, ducharse, hacer tareas, etc., donde \\
LA ACTIVIDAD & los niños deben reconocer la cronología adecuada de la actividad siempre enfocados en la \\
& importancia del cuidado de la salud propia por medio de la adecuación de hábitos de vida \\
& saludable. Un ejemplo de simulación es durante la ducha: se presentan las tarjetas con las \\
& actividades en desorden y los niños deben ordenarlas y simular la actividad de la tarjeta: \\
& a) quitarse la ropa, b) abrir la llave y mojarse, c) cerrar llave y aplicar jabón y shampoo, d), \\
enjuagarse en poco tiempo, e) secarse solo y f) salir del baño y vestirse; las actividades estaban & en desorden y ellos deben organizarlas también creando conciencia del cuidado del medio \\
ambiente.
\end{tabular}

Fuente: elaboración propia (2016)

Tabla 4. "Soy un rompecabezas"

\begin{tabular}{ll}
\hline ALCANCE & Promoción \\
\hline ACTIVIDAD & Soy un rompecabezas \\
\hline OBJETIVO & $\begin{array}{l}\text { Potenciar el reconocimiento del esquema corporal, a través de patrones específicos que } \\
\text { segmenten el cuerpo en términos de actividades y funciones. }\end{array}$ \\
\hline DESCRIPCIÓN DE & Se realiza en dos tiempos, el primero se divide el grupo en 2 a través de manillas de colores, \\
LA ACTIVIDAD & se realizan diferentes actividades como carrera de gateo, saltos en un solo pie en diferentes \\
& texturas, rolados, botes y carreras persiguiendo objetos, durante la actividad se retroalimenta \\
& a los niños para que reconozcan la utilidad de sus manos, pies y rodillas, reconociéndolas \\
& como partes de su propio cuerpo que les son útiles para interactuar en su entorno. \\
\hline RESULTADOS & Se busca la identificación por parte de los niños de las partes de su cuerpo, reconociendo \\
las diferencias entre los niños y las niñas y lo más importante es que se espera la integración \\
del esquema corporal, identificando sus partes funcionales y las posibilidades de interacción \\
en el mundo.
\end{tabular}

Fuente: elaboración propia (2016)

Tabla 5. "Siendo beisbolistas"

\begin{tabular}{ll}
\hline ALCANCE & Promoción \\
\hline ACTIVIDAD & Siendo beisbolistas \\
\hline OBJETIVO & $\begin{array}{l}\text { Identificar las habilidades, capacidades y destrezas de la ejecución motora en el béisbol, dando } \\
\text { cuenta del mejor patrón desempeñado por el niño, ya sea batear o lanzar, lo anterior con el } \\
\text { fin de definir un rol en el juego }\end{array}$ \\
\hline
\end{tabular}


DESCRIPCIÓN DE LA ACTIVIDAD
Se escoge el béisbol debido a que este deporte permite desarrollar un adecuado proceso de aprendizaje del patrón maduro de lanzamiento y contribuye a mejorar coordinación ojo-mano. La actividad se desarrolla en 3 momentos, el primero de calentamiento, el segundo de aprendizaje de patrones del béisbol y el tercero de jugarlo con las reglas. El primero se realiza a la vez con todo el grupo, en el segundo se dividen en 2 grupos los que batean y los que lanzan la pelota se realizan ejemplo de cómo se debe hacer aprovechando el aprendizaje por imitación de los niños y el último se realiza por parejas y posteriormente por equipos para aprender las reglas y que disfruten de esa nueva experiencia a medida que reconocen el deporte como hábito de vida saludable.

RESULTADOS ESPERADOS Se espera que los niños reconozcan el deporte como hábito saludable. Asimismo, cada uno identificó cuál es la posición que mejor desempeña, a su vez se evidencia el carácter competitivo, aprendiendo que no solo es importante ser el más rápido, sino que realicen la actividad con el mejor performance.

Fuente: elaboración propia (2016)

\section{ACTIVIDADES PARA NIÑOS ENTRE 6 A 8 AÑOS}

Tabla 6. "Mis destrezas en un Rally"

\begin{tabular}{ll}
\hline ALCANCE & Promoción \\
\hline ACTIVIDAD & Mis destrezas en un rally \\
\hline OBJETIVO & $\begin{array}{l}\text { Generar un proceso de aprendizaje acerca de hábitos de vida saludable a través de actividades } \\
\text { en las que conozcan rutinas de autocuidado para que con el tiempo esto sea parte de su vida } \\
\text { diaria. }\end{array}$ \\
\hline $\begin{array}{l}\text { DESCRIPCIÓN DE } \\
\text { LA ACTIVIDAD }\end{array}$ & $\begin{array}{l}\text { La actividad consiste en una carrera de obstáculos, donde hay diferentes actividades de } \\
\text { competencia como: saltos unipodales con movimientos alternados de miembros superiores } \\
\text { e inferiores (en caballito), carretilla, carrera saltando aros, carreras pasando por encima y } \\
\text { debajo de obstáculos, saltos en un solo pie, cangrejos. Gana el que lo hace de forma correcta } \\
\text { no el más rápido. }\end{array}$ \\
\hline RESULTADOS & $\begin{array}{l}\text { La actividad pretende despertar en los niños el interés por realizar actividad física, por medio } \\
\text { de carreras de obstáculos, adicionalmente incentivar la sana competencia. }\end{array}$ \\
\hline
\end{tabular}

Fuente: elaboración propia (2016)

Tabla 7. "El semáforo de la alimentación"

\begin{tabular}{ll}
\hline ALCANCE & Promoción y prevención \\
\hline ACTIVIDAD & El semáforo de la alimentación \\
\hline OBJETIVO & $\begin{array}{l}\text { Promover hábitos de vida saludable donde los niños reconozcan la necesidad de mantener } \\
\text { una dieta saludable con el consumo de diferentes nutrientes por medio de métodos didácticos, } \\
\text { conociendo sus aportes y beneficios de la regulación nutricional para la realización de actividades. }\end{array}$ \\
\hline DESCRIPCIÓN DE & $\begin{array}{l}\text { Se debe realizar una introducción acerca de los hábitos alimenticios. Posterior a esto se } \\
\text { formará un círculo, en el medio de este habrá una pirámide y alrededor y en desorden estarán } \\
\text { ubicadas imágenes de alimentos como lo son los encontrados en el desayuno, almuerzo, cena } \\
\text { y comida chatarra. } \\
\text { Los niños deberán reconocer la pirámide nutricional, de modo que puedan caracterizar cada } \\
\text { alimento en función de su relación con los efectos en el cuerpo para las actividades de la vida diaria. } \\
\text { Los colores del semáforo permiten categorizar una adecuada, media o inadecuada selección } \\
\text { de los alimentos. }\end{array}$ \\
\hline
\end{tabular}




\section{RESULTADOS ESPERADOS}

Se logró la adquisición de conocimiento significativo sobre la importancia de una alimentación balanceada y comprendieron su influencia sobre la adquisición de energía para realizar actividad física.

Fuente: elaboración propia (2016)

Tabla 8. "Fiesta hawaiana"

\begin{tabular}{ll}
\hline ALCANCE & Promoción y prevención \\
\hline ACTIVIDAD & Fiesta hawaiana \\
\hline OBJETIVO & $\begin{array}{l}\text { Potenciar el desarrollo de patrones fundamentales a partir de actividades de motricidad fina } \\
\text { fomentando la creatividad y el seguimiento de instrucciones de manera secuencial. }\end{array}$ \\
\hline DESCRIPCIÓN DE & $\begin{array}{l}\text { Elaboración de accesorios. } \\
\text { LA ACTIVIDAD }\end{array}$ \\
& Se mostrará un instructivo gráfico con los pasos a seguir. Posteriormente se entregan los \\
& materiales para la realización de un collar y una manilla en la cual se utilizaran pitillos, flores \\
& donde los niños iniciaran a hacer sus implementos cada uno con sus materiales siguiendo las \\
& instrucciones dadas las cuales consisten en ensartar nylon en un trozo de pitillo, luego una flor \\
& y luego otro trozo de pitillo así sucesivamente hasta completar el collar, luego se realizará un \\
& nudo para que no se salgan los materiales; al finalizar el collar se hará el mismo procedimiento \\
& para la elaboración de las manillas; para terminar se retroalimenta la actividad observando \\
& los implementos realizados por cada uno de los niños y se realiza una socialización final sobre \\
la experiencia motriz y la experiencia creativa.
\end{tabular}

RESULTADOS ESPERADOS Se busca que por medio de acciones motrices manuales los niños reconozcan su capacidad de innovar mostrando su creatividad y expresándose libremente.

Fuente: elaboración propia (2016)

Tabla 9. "Cuidando mis dientes"

\begin{tabular}{ll}
\hline ALCANCE & Promoción y prevención \\
\hline ACTIVIDAD & Higiene personal \\
\hline OBJETIVO & $\begin{array}{l}\text { Promover el aprendizaje motor a través de actividades de autocuidado bucal, como situación } \\
\text { clave en contextos de la vida diaria. }\end{array}$ \\
\hline DESCRIPCIÓN DE & $\begin{array}{l}\text { La actividad de inicio consistirá en un socio drama donde los niños representarán los principales } \\
\text { Lomentos de cepillado de dientes y porque son los adecuados para este, como por ejemplo } \\
\text { después de cada comida, al levantarse o antes de ir a dormir. Y luego que expliquen lo que } \\
\text { sucede cuando no se cepillan sus dientes. } \\
\text { Como actividad central se inicia con la presentación de los dientes uno sano y fuerte y el otro } \\
\text { con caries y sucio, se procederá a cepillarse los dientes siguiendo las instrucciones dadas por } \\
\text { los estudiantes. } \\
\text { Como actividad final consistirá en la construcción de un folleto cada niño donde dibujaran } \\
\text { la secuencia de un cepillado adecuado de dientes junto con las veces en las cuales se deben } \\
\text { cepillar los dientes cada niño. }\end{array}$ \\
\hline RESULTADOS ESPERADOS & $\begin{array}{l}\text { Se espera que los niños generen autoconciencia sobre el autocuidado a partir de un adecuado } \\
\text { cepillado de los dientes identificando las diferentes complicaciones que puede conllevar y de } \\
\text { la importancia que tiene para su cuerpo cuidarse en distintos aspectos como en el cepillado } \\
\text { de dientes. }\end{array}$ \\
\hline
\end{tabular}

Fuente: elaboración propia (2016) 


\section{ACTIVIDADES PARA NIÑOS ENTRE 9 A 14 AÑOS}

Tabla 10. "Carrera dental"

\begin{tabular}{ll}
\hline ALCANCE & Promoción y prevención \\
\hline ACTIVIDAD & Carrera dental \\
\hline OBJETIVO & $\begin{array}{l}\text { Estimular la motricidad gruesa en actividades de competencia en una carrera de obstáculos } \\
\text { centrada en prácticas de autocuidado (higiene oral, hábitos y rutinas saludables), favoreciendo } \\
\text { el auto concepto y la independencia en actividades de la vida diaria. }\end{array}$ \\
\hline DESCRIPCIÓN DE & Se forman 3 grupos de 5 niños cada uno. \\
LA ACTIVIDAD & Teniendo en cuenta que se tiene previo conocimiento sobre los procesos de higiene oral la \\
& actividad consiste en responder una serie de preguntas orientadoras alusivas a la higiene bucal, \\
& estas preguntas se distribuyen por lugares específicos de la institución y deben buscarlas por \\
& medio de unas pistas entregadas al inicio, una vez se da la respuesta a todas las preguntas \\
deben llegar al punto de inicio para retroalimentar y plasmar en un mural lo aprendido con & esta actividad; Finalmente se entrega un reconocimiento que es pegado en el mural llamado \\
"el rincón de la salud".
\end{tabular}

Fuente: elaboración propia (2016)

Tabla 11. "Un día en el gimnasio "

\begin{tabular}{ll}
\hline ALCANCE & Promoción \\
\hline ACTIVIDAD & Un día en el gimnasio \\
\hline OBJETIVO & $\begin{array}{l}\text { Explorar las capacidades expresivas y motrices a partir de estrategias como rumba, aeróbicos } \\
\text { y entrenamiento funcional, que permitan promover el interés por la práctica de actividad } \\
\text { física en los niños. }\end{array}$ \\
\hline $\begin{array}{l}\text { DESCRIPCIÓN DE LA } \\
\text { ACTIVIDAD }\end{array}$ & $\begin{array}{l}\text { Se inicia con una fase de calentamiento con aeróbicos, posteriormente en la fase central se } \\
\text { realizan ejercicios con los métodos de entrenamiento de CORE y entrenamiento funcional } \\
\text { finalizando la actividad se realiza una sesión de relajación muscular, por medio de estiramientos } \\
\text { activos con duración de } 20 \text { segundos por grupo muscular. }\end{array}$ \\
\hline RESULTADOS & $\begin{array}{l}\text { Se espera que los niños identifiquen la importancia de hacer de la actividad física parte de sus } \\
\text { hábitos cotidianos, por medio de la concepción de esta como un hábito "divertido" generando } \\
\text { mayor conocimiento sobre las diferentes alternativas para realizarla. }\end{array}$ \\
\hline
\end{tabular}

Fuente: elaboración propia (2016)

Tabla 12. "Rumba latina"

\begin{tabular}{ll}
\hline ALCANCE & Promoción \\
\hline ACTIVIDAD & Rumba latina \\
\hline OBJETIVO & Fortalecer la participación a través de actividades artísticas manuales (creación de disfraces) y \\
& de expresión corporal que permitan poner en práctica el potencial creativo y de comunicación \\
& no verbal de los niños. \\
\hline
\end{tabular}


DESCRIPCIÓN DE LA ACTIVIDAD
Puesto que esta actividad es complementaria de la fiesta hawaiana realizada con los niños de 1 a 5, la actividad inicia con la elaboración de la indumentaria: collares, manillas y demás accesorios referentes al tema de la fiesta.

Posteriormente se realiza una actividad enfocada en el libre desarrollo de la expresión corporal a través del baile, para la cual se realiza una previa selección de música de interés para los niños, a partir de la cual se da la indicación de representar el ritmo de la música expresando habilidades ya sea individuales o grupales sobre el baile.

RESULTADOS ESPERADOS Se pretende que a través de la realización del taller se genere en el grupo motivación para desarrollar un óptimo trabajo colectivo y que a través de sus habilidades de creación e imaginación logren plasmar sus gustos y pensamientos, utilizando su cuerpo como herramienta principal de expresión que permita compartir sus ideas con los demás.

Fuente: elaboración propia (2016)

Tabla 13. "Escalera dinámica"

\begin{tabular}{|c|c|}
\hline ALCANCE & Promoción \\
\hline ACTIVIDAD & Escalera dinámica \\
\hline OBJETIVO & $\begin{array}{l}\text { Favorecer la práctica de actividad física como un recurso para el aprovechamiento del tiempo } \\
\text { libre, por medio de la implementación de actividades recreativas, con el fin de reforzar este } \\
\text { hábito saludable. }\end{array}$ \\
\hline \multirow[t]{2}{*}{$\begin{array}{l}\text { DESCRIPCIÓN DE } \\
\text { LA ACTIVIDAD }\end{array}$} & $\begin{array}{l}\text { Se recrea un tablero que simula el juego de escalera en tamaño real donde cada jugador } \\
\text { inicia desde la casilla de partida, por turnos cada jugador lanza un dado y avanza el número } \\
\text { que indique el dado, las casillas estarán identificadas con colores que indicarán una serie de } \\
\text { actividades enfocadas hacia el ejercicio. Las actividades son: }\end{array}$ \\
\hline & $\begin{array}{l}\text { - Saltar a lazo (aumentan las repeticiones a medida que se acerca a la meta, hasta un máximo } \\
\text { de } 15 \text { repeticiones) } \\
\text { - Hacer sentadillas (cuclillas) } \\
\text { - Abdominales } \\
\text { - Alcanzar la punta de los pies sin doblar las rodillas } \\
\text { - Acostarse y hacer "bicicleta" } \\
\text { - Realizar cualquier movimiento corporal al ritmo de una canción colocada por los terapeutas. }\end{array}$ \\
\hline RESULTADOS ESPERADOS & $\begin{array}{l}\text { Esta actividad permite fomentar en los niños el interés por la actividad física y el reconocimiento } \\
\text { de esta como parte fundamental para tener un estilo de vida saludable, logrando que apliquen } \\
\text { las recomendaciones aprendidas a la vida cotidiana. }\end{array}$ \\
\hline
\end{tabular}

Fuente: elaboración propia (2016)

\section{DISCUSIÓN}

Colombia cuenta con normativas vigentes y bases legales vinculadas con la población infantil, como la política pública de Cero a Siempre, el Código de Infancia y Adolescencia, la Constitución Política de Colombia, Ley 181 de 1995 y Plan Decenal del Deporte, la Recreación, la Educación Física y la Actividad Física, para el Desarrollo Humano, la Convivencia y la Paz 2009-2019; las cuales cimientan la propuesta de este documento.
La estrategia de Cero a Siempre se enfoca en mujeres gestantes, niñas y niños; proponiendo un conjunto de acciones planificadas que tienen el objetivo de promover y garantizar el desarrollo infantil de las niñas y los niños desde su gestación hasta cumplir los seis años; se realiza por medio de un trabajo unificado e intersectorial que desde la perspectiva de derechos y con un enfoque diferencial, articula y promueve el desarrollo de planes, programas, proyectos y acciones para la atención integral que debe asegurarse a cada 
niña y niño, de acuerdo con su edad, contexto y condición (Presidencia de la República, 2013).

Lo anterior, se articula con el Código de Infancia y Adolescencia, según el cual, desde la Ley 1098 de 2006, se establece que se debe garantizar el pleno cumplimiento de cada uno de los derechos de los niños, niñas y adolescentes, siendo obligación de los actores tales como familia, sociedad y estado.

A partir de la Constitución Política de 1991 de Colombia en el artículo 44 se establece que:

Son derechos fundamentales de los niños: la vida, la integridad fisica, la salud y la seguridad social, la alimentación equilibrada, su nombre y nacionalidad, tener una familia y no ser separados de ella, el cuidado y el amor, la educación y la cultura, la recreación y la libre expresión de su opinión. Serán protegidos contra toda forma de abandono, violencia física o moral, secuestro, venta, abuso sexual, explotación laboral o económica y trabajos riesgosos. Gozarán también de los demás derechos consagrados en la Constitución, en las leyes y en los tratados internacionales ratificados por Colombia. (Constitución Política de Colombia, 1991, p. 15).

Teniendo en cuenta los derechos de los niños, respaldados en las normativas anteriores y las necesidades básicas reconocidas específicamente en aquellos niños en situación de protección, se tomó como eje central desde la Fisioterapia la limitación en cuanto a la disponibilidad de actividades relacionadas con el movimiento, programadas y diferenciadas por etapas del ciclo vital.

En este sentido, el Sistema Nacional de Deportes, creado por la Ley 181 de 1995 y el Plan Decenal del Deporte, la Recreación, la Educación Física y la Actividad Física, para el Desarrollo Humano, la Convivencia y la Paz 2009-2019, aporta a la promoción de la actividad física puesto que tiene como objetivo principal patrocinar y masificar la práctica de actividades deportivas, el aprovechamiento del tiempo libre, la instauración de espacios recreativos, donde la mayoría de la población pueda participar de forma activa teniendo en cuenta principalmente a los niños, adolescentes y personas en condición de discapacidad; haciendo énfasis en la promoción de la educación extraescolar, para que desde edades tempranas los niños participen de estos espacios y sea más viable convertir en hábito la práctica de estas actividades o deportes, promoviendo una cultura y estilos de vida saludable.

Ahora bien, las actividades propuestas en este documento además de estar respaldadas por la legislación actual, se soportaron conceptualmente desde las etapas del ciclo vital, teniendo en cuenta las particularidades del desarrollo motor.

A partir de esto, el diseño de actividades para las edades de 1 a 5 años se dirigieron principalmente al desarrollo de patrones básicos fundamentales y refuerzo del esquema corporal, esto es consecuente con los hallazgos de la evaluación, específicamente en la evaluación general de la EAD, en donde el desarrollo de los niños no se encontraban acorde a su edad cronológica.

En las edades de 6 a 8 años se buscó implementar actividades dirigidas a la potencialización y mantenimiento de los patrones fundamentales adquiridos y el reconocimiento de los aportes y beneficios del consumo de nutrientes, este último más como componente esencial para la programación de actividad física que como componente sensible en la evaluación realizada.

En las edades de 9 a 14 años se buscó fomentar actividades que generen un impacto significativo sobre el aprendizaje y el desarrollo de destrezas, habilidades y herramientas, que garanticen un estilo de vida saludable durante su participación en la sociedad. Además, dentro de las actividades planteadas se incorporaron temas a trabajar de forma transversal en los tres grupos etarios, como lo fueron el aprovechamiento del tiempo libre, estrategias de autocuidado y la participación activa dentro de un deporte.

En concordancia con lo descrito anteriormente, se justifican las actividades a partir de los alcances del movimiento corporal humano sobre el rol específico que desempeñan los niños desde cada rango etario. 
A partir de esto se encuentra que en las edades de 1 a 5 años es de gran importancia estimular la práctica de patrones motores fundamentales teniendo en cuenta el desarrollo a nivel neurológico, en términos de reflejos y reacciones que le permitirán tener adecuado uso y dominancia de sus habilidades y capacidades que potencien la ejecución de los patrones básicos de movimiento (Álvarez, 2012).

Otro de los aspectos a trabajar en este rango etario es el desarrollo del esquema corporal, ya que es fundamental para los niños reconocer las partes de su cuerpo inicialmente como segmentos, luego como un todo y posteriormente utilizarlo como herramienta que permita interactuar con su entorno próximo (Gallego del Castillo, 2010). No hay que dejar de lado que el esquema corporal se relaciona con el auto concepto físico, esto permite reconocer la presencia de sus habilidades referentes a las partes de su cuerpo y la función de estas (Caballero, 2006).

En las edades de 6 a 8 años las actividades van encaminadas a que los niños después de adoptar patrones locomotores (que vinculan motricidad fina y gruesa) logren mejorarlos y realizarlos de una manera óptima; estos patrones se desarrollan de manera diferente en cada niño, pero tienen el mismo orden y unas características las cuales permiten adaptarse al medio respondiendo de forma asertiva y eficiente a la exigencia requerida. (Calero y García, 2014).

En las edades de 8 a 14 años es de gran importancia estimular la práctica de un estilo de vida saludable a través de la potencialización de habilidades psicomotoras que a su vez permitan la máxima satisfacción en todas las etapas de la vida y durante su interacción en los diferentes entornos.

Lo anterior, teniendo en cuenta que los niños interactúan por medio de sus capacidades actuales para obtener como resultado una consolidación y construcción de nuevas habilidades (Amador, 2013); esto a su vez les permite actuar de manera competente en distintas situaciones de la cotidianidad reforzando el desarrollo de comportamientos saludables y adicionalmente mantener un control de su vida en interacción con el medio
(Morales, Benítez y Agustín, 2013); esto, teniendo en cuenta que es la edad más próxima a la salida de las instituciones y a partir de allí es importante estipular bases para el manejo de la vida cotidiana.

Se considera pertinente desarrollar actividades que favorezcan el aprovechamiento del tiempo libre, estrategias de autocuidado y participación activa dentro de algún deporte que genere un vínculo entre las personas de cada uno de los rangos etarios.

Esto fue un componente clave dentro de la proyección de esta propuesta en la medida en que se logró identificar aquellas actividades con las cuales los niños se sentían más identificados y aquellas en las cuales tienen más habilidad. Además, la articulación con actividades de autocuidado permite que se generen procesos de asociación entre el adecuado manejo del tiempo y la adquisición de hábitos relacionados directamente con la vida saludable, permitiendo que el sujeto desarrolle su autonomía por medio de la práctica y el aprendizaje (Ministerio de Salud, 2014).

Dentro de esto, la actividad física permite el incremento de la autoestima, mejora la salud y previene lesiones musculoesqueléticas, además, enriquece la vida social y el desarrollo y recuperación de capacidades físicas y mentales, estimulando también el coeficiente de los niños, niñas y jóvenes, por otra parte, el refuerzo en la psicomotricidad es fundamental en el desarrollo de los aspectos que influyen en la personalidad del niño como son el cognitivo, afectivo, social y motor, mediante lo cual reconocen su cuerpo y a través de este interactúan en el mundo que los rodea (Bilbao, Corres y Urdampilleta, 2012).

Es necesario que dentro de un proceso basado en actividad física, que la población objeto (en este caso los niños), reconozcan la importancia de los aportes y beneficios del consumo de nutrientes debido a que durante la niñez es importante una alimentación balanceada y adecuada acompañada de una práctica de actividad física para tener un correcto consumo-gasto y de esta manera evitar el desarrollo de enfermedades no transmisibles principalmente la obesidad la cual según la OMS (2008) ha aumentado a nivel mundial en niños 
(Sanchez-Garcia, Reyes-Morales y González-Unzaga, 2014), por lo cual las actividades van encaminadas a mantener y reconocer unos hábitos alimenticios óptimos.

\section{CONCLUSIONES}

Este documento propone de forma estructurada presentar una estrategia con potencial de beneficio práctico y con capacidad de extenderse a diferentes instituciones que realicen atención a niños en situación de protección.

Una de las finalidades de la estrategia, se basó en que los procesos de intervención realizados por los profesionales en Fisioterapia sean continuos y significativos en los procesos de aprendizaje y desarrollo de la población en general, acompañado de indicaciones para los actores que intervienen directamente con los niños y de esta manera cuenten con las herramientas para realizar intervención continua de la estrategia. Así mismo, es importante que se reconozca la importancia del rol del fisioterapeuta y cómo este aporta al desarrollo global en la primera infancia y a lo largo del ciclo vital.

\section{AGRADECIMIENTOS}

El presente artículo fue realizado bajo la dirección del docente Carlos Alberto Pérez Gómez.

\section{REFERENCIAS}

Álvarez, L. (2012). Evaluación Psicomotriz a niños y niñas con trastorno del Espectro Autista desde la Perspectiva de la Psicomotricidad Vivenciada. Recuperado de: http://ridum.umanizales.edu. co:8080/xmlui/bitstream/handle/6789/261/alvarez_toro_ lina_johana-2012.pdf?sequence $=1$

Amador, E. (2013). Las habilidades motoras en niños víctimas de maltrato físico. Revista Salud Uninorte. 29(2), 280287. Recuperado de: http://www.scielo.org.co/scielo. php?script $=$ sci_arttext\&pid $=$ S0120-55522013000200013

Bellamy, C. (2005). Estado Mundial de la Infancia. La Infancia Amenazada. Fondo de las Naciones Unidas para la Infancia. Recuperado de: http://www.unicef.org/spanish/sowc05/ sowc05_sp.pdf

Bilbao, L; Corres, U y Urdampilleta, A. (2012). La importancia de la psicomotricidad en la actividad físico-deportiva extraescolar. EFDeportes.com Revista Digital. 16(165). Recuperado de: http://www.efdeportes.com/efd165/laimportancia-de-la-psicomotricidad.htm

Caballero, L. (2006). Autoconocimiento. Junta de Andalucía. Recuperado de: http://www.portalempleofemm.org/pdf/ guia_autoconocimiento.pdf

Calero, P. y García, H. (2014). Perfil psicomotor de los niños en edades entre 6 y 10 años, pertenecientes a los planteles educativos de la ciudad de Pereira. Universidad Autónoma de Manizales, Facultad de Salud. Pereira.

Cigarroa I, Sarqui C, Zapata-Lamana R. (2016). Efectos del sedentarismo y obesidad en el desarrollo psicomotor en niños y niñas: Una revisión de la actualidad latinoamericana. Revista Univ. Salud. 18(1), 156-169. Recuperado de: http://www.scielo.org.co/pdf/reus/v18n1/ v18n1a15.pdf

Constitución Política de Colombia. [Const.] (1991) 2da Ed. Legis.

Congreso de Colombia. (18 de enero de 1995). Ley 181 de 1995. DO: 41.679 Recuperado de: http://www.mineducacion. gov.co/1621/articles_104567_archivo_pdf.pdf

Congreso de Colombia. (14 de septiembre de 1999) Ley 528 de 1999. DO: 43.711. Recuperado de: http://www. mineducacion.gov.co/1621/articles-105013_archivo_pdf. pdf

Congreso de Colombia. (8 de noviembre de 2006). Código de la Infancia y la Adolescencia. [Ley 1098 de 2006]. DO: 46446. Recuperado de: http://www.mineducacion.gov. co/1621/articles_104567_archivo_pdf.pdf

Gallego del Castillo, F. (2010). Esquema Corporal y Praxia. Bases conceptuales. España. Wanceulen Editorial deportiva.

Herazo, Y., Hernández, J. y Domínguez, R. (2012). Etapas de cambio y Niveles de Actividad Física en estudiantes Universitarios de Cartagena (Colombia). Revista científica Salud Uninorte. 28(2). Recuperado en: http://rcientificas. uninorte.edu.co/index.php/salud/articleview/1168/3955

Hernández, R., Fernández, C. y Baptista, P. (2014). Metodología de Investigación. 6ta Ed. México: Mc Graw Hill.

Martínez-Gómez, T. et al. (2009). Fiabilidad y Validez del Cuestionario de Actividad Física PAQ-A en adolescentes españoles. Revista Española Salud Pública. 83(3), 427-439.

Ministerio de Salud (2014). Guía Básica Higiene y Cuidado del Cuerpo. Recuperado en: http://salud.mendoza.gov.ar/ wp-content/uploads/sites/16/2014/09/higiene_cuidado_ cuerpo.pdf

Morales, M; Benítez, M y Agustín, D. (2013). Habilidades para la vida (cognitivas y sociales) en adolescentes de zona rural. Revista Electrónica de Investigación Educativa. 
15(3). Recuperado de: http://www.scielo.org.mx/scielo. php?script $=$ sci_arttext\&pid=S1607-40412013000300007

Muñoz, A; Zapata-Ossa, H. y Pérez- Tenorio, L. (2013). Validación de criterio de la Escala Abreviada del Desarrollo (EAD-1) en el dominio Audición Lenguaje. Revista de Salud Pública. 15(3), 386-397. Recuperado de: http://revistas.unal.edu. co/index.php/revsaludpublica/article/view/36640/44549

OCDE. (2003). Manual de Frascati. Propuesta de norma práctica para encuestas de investigación y desarrollo experimental. Organización para la cooperación y el desarrollo económico (OCDE), Fundación Española para la Ciencia y la Tecnología (FECYT). Recuperado de http:// www.id.mineco.gob.es/stfls/MICINN/Investigacion/ FICHEROS/ManualFrascati-2002_sp.pdf

OMS. (2010). Recomendaciones Mundiales sobre Actividad Física para la Salud. Recuperado de: http://apps.who.int/iris/ bitstream/10665/44441/1/9789243599977_spa.pdf

OMS. (2013). El desarrollo del niño en la primera infancia y la discapacidad: Un documento de debate. Recuperado de: https:// www.unicef.org/earlychildhood/files/ECDD_SPANISHFINAL_(low_res).pdf

Presidencia de la República. (2013). Estrategia de atención Integral a la Primera Infancia. Fundamentos Políticos, Técnicos y de Gestión de cero a siempre. Recuperado en: http://www.deceroasiempre.gov.co/QuienesSomos/ Documents/Fundamientos-politicos-tecnicos-gestionde-cero-a-siempre.pdf

Presutti, F. (s.f). Modelos y Estrategias Docentes en la Educación de Infantil. Universidad de Monterrey. En:http://www.ispef.
org/Monterrey2010/Temas\%20de\%20el\%20Corso $\% 20$ di\%20Monterrey/Cap.7\%20\%20Perfil\%20y\%20roles\%20 \%20pag.\%2078-87\%20doc.\%20(sp).pdf

República de Colombia y cols. (2009). Plan Decenal del Deporte, la Recreación, la Educación Física y la Actividad Física, para el Desarrollo Humano, la Convivencia y la Paz, 2009-2019. Recuperado de: http://www.coldeportes. gov.co/testing_new/index.php?idcategoria $=57928 \& d$ ownload $=\mathrm{Y}$

Sánchez-García, R. Reyes-Morales, H. y González-Unzaga, M. (2014). Preferencias alimentarias y estado de nutrición en niños escolares de la Ciudad de México. Bol Med Hosp Infant Mex. 71(6), 358-366.

UNICEF, (2005). La Niñez Colombiana en Cifras. Recuperado el 8 de diciembre de 2016 en: https://www.unicef.org/ colombia/pdf/cifras.pdf

Vargas, I., Villegas, O., Sánchez, A. y Holthuis, K. (2003). Promoción y Prevención y Educación para la Salud. Primera Edición. San José de Costa Rica. Recuperado de: http:// www.montevideo.gub.uy/sites/default/files/concurso/ documentos/Ed211.paralaSalud-MariaT.Cerqueira.pdf

Vignolo, J., Vacarezza, M., Álvarez, C. y Sosa, A. (2011). Niveles de atención, prevención y atención primaria de la Salud. Archivos de Medicina Interna. 33(1), 1114.

Zamora, J. y Laclé, A. (2012). Evaluación del gasto energético y actividad física en escolares eutróficos, con sobrepeso y obesidad. Revista Chilena de Pediatría. 83(2), 134-145. 